\title{
Research on the application of target detection based on deep learning technology in power grid operation inspection
}

\author{
Peng Weifu ${ }^{1}$, Du Shu ${ }^{2}$, Chen Shaolei ${ }^{3}$, Zhou Qing ${ }^{4}$, Tang $\mathrm{Na}^{5}$ \\ ${ }^{1}$ Information and communication transportation Inspection Center, State Grid Sichuan Information \&telecommunication Company, \\ Sichuan, China \\ ${ }^{2}$ Information and communication transportation Inspection Center, State Grid Sichuan Information \&telecommunication Company, \\ Sichuan, China \\ ${ }^{3}$ Power dispatching control center, State Grid Sichuan Electric Power Company, Sichuan, China \\ ${ }^{4}$ Research and development, Whayer Intelligent Technology Group Co., Ltd. Sichuan, China \\ ${ }^{5}$ Research and development, Sichuan GRID Power Communication Technology Co., Ltd. Sichuan, China
}

\begin{abstract}
External damage to power facilities caused by crane, excavator and other construction operations increases year by year, which will seriously threaten the safe operation of power system. It is an important measure to ensure the safe and reliable operation of power system to implement intelligent monitoring and early warning of power external breakdown through video and other non-contact observation means. The video data of power mainly comes from the fixed monitoring of helicopters, uavs and transformation poles and towers, which is characterized by large amount of data, complex scenes and serious environmental interference. The traditional target detection method usually selects the candidate area first, and then makes judgment based on the characteristics of human construction. The detection speed is slow and the accuracy is low, which makes it impossible to monitor the video data in real time, so as to make timely and accurate early warning and intervention fbr external damage. The target detection method based on deep learning optimizes or even eliminates the selection of candidate regions, which greatly speeds up the detection speed. By learning a lot of target samples through the deep neural network, the characteristics of high robustness are gradually fitted to make the target judgment more accurate.

There are three key problems in introducing the target detection method based on deep learning into the power video detection: Firstly, the target detection method based on deep learning has a large amount of calculation and many parameters. In order to realize in-place operation on terminals with limited computing and storage capacity, it is necessary to find a practical method to simplify the network and reduce the amount of operational data in the detection process, which is the key to realize in-place operation and terminal operation of deep neural network. Secondly, for specific application scenarios, the effect of different target detection algorithms varies greatly, and there is a strong particularity of power video. Finding an effective target detection method is the key to improve the detection speed and accuracy. Finally, with the continuous development of deep learning, the structure of deep neural network changes with each passing day, and each has its own characteristics, which network structure is used as the feature extraction layer of target detection algorithm is the focus of research.
\end{abstract}

\section{Introduction}

\subsection{Subject background, purpose and significance of the research}

Video data of power system usually adopts the method of first collecting, then summarizing, and finally unified processing in the cloud (data center, server cluster). In this mode, all pictures and videos need to be transmitted to the cloud through network or storage devices for processing, which greatly increases the burden of data storage. In addition, The judgment process requires steps such as image and video collection, cloud transmission, cloud processing, and return of judgment results, with high delay and poor real-time detection. In addition, the cloud transmission process is likely to cause data leakage, so data security cannot be guaranteed. Therefore, it is of great significance to realize the local and terminal operation of the target detection system. The target detection method based on deep learning is difficult to realize terminal transplantation due to its large computational load and numerous parameters. Finding a feasible method to simplify the network and reduce the amount of operational data in the detection process is the key to realize the localization of deep neural network and terminal operation. Neural network trained by compression algorithm makes the deep learning algorithms of neural network model can 
be implemented in the special chip in a very low power consumption, the requirements of low power consumption embedded node side can also have "machine vision" "independent discriminant" intelligent "function", because the judgment in embedded devices directly, without the data of intensive processing, can realize online judgment, effectively solve the problems of existing patterns.

\section{Power vision terminal target detection system}

\subsection{Hardware of the system}

In terms of hardware, Swift series deep learning processor products are presented in the form of PCB board card, as shown in Figure 2-1. The board card is divided into two layers, with length and width of $50 \mathrm{~mm} * 50 \mathrm{~mm}$ and height of $30 \mathrm{~mm}$. Among them, board card 1 places Swift series deep learning processor, ARMA9 processor, 1GBDDR storage particles and other core devices, while board card 2 places external interface, power supply, etc. The external interface class is customized according to user requirements. The list of interfaces is shown in Table 2-1.

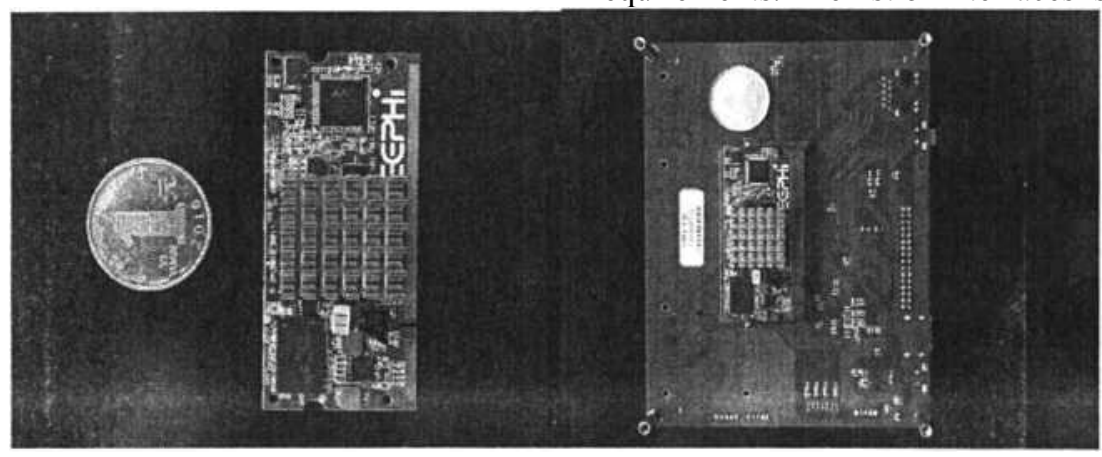

Figure 2-1 EMBEDDED TERMINAL CNN product Swift

Compared with the mobile GPU with similar positioning, the platform chooses the most representative NVIDIATegraKlmGPU as the benchmark platform, and makes a comparison with the convolutional neural network of VGG-16. As shown in Table 3-3, the power consumption of DPU is about $1 / 5-1 / 6$ that of mGPU, while the computing speed is nearly twice that of mGPU. In addition, the DPU processor is equipped with the corresponding compilation software, which seamlessly connects with the neural network model under the standard framework. The software can automatically complete the process of network structure compression, fixed-point and compilation, so that the neural network algorithm design can be effectively implemented in the embedded end with one key.

\subsection{System software}

Combined with compression and compilation technology and hardware structure design, the DPU processor can achieve the convolution computing speed of $173 \mathrm{GOPS}$ and the global computing speed of 102GOPS when processing CNNlike tasks and realizing the lossless recognition degree of Imagenet image classification scene (VGG16, TOP5 accuracy 86.67\%). Compared with the platforms of CPU and GPU, the power consumption of DPU is only $3.66 \mathrm{~W}$ compared with the high power consumption of CPU and GPU. In terms of energy efficiency ratio, DPU is 4-38 times higher than CPU, GPU and mobile GPU, which is very suitable for deployment in mobile terminal.

\subsection{Deep compression quantization of convolutional neural network}

This paper adopted a pruning, quantification and Huffman coding compression method, the depth of the neural network the method from the international field of deep learning ICLR2016 paper of the year's top meeting Deep Compression: Compressing Deep Neural Networks With Pruning Trained Quan tization And Huffman Coding ", can be without degradation in detection precision for dozens of times, compression ratio figure 2-2 whole working process of the method is presented. The method mainly consists of three steps. First, by retraining the network model, the nodes and connections that are relatively "important" in the model connection are screened out, and the nodes and connections that are relatively unimportant are clipped, as shown in Figure 23. Secondly, the weight parameters are clustered to generate codebook, and the weight is quantified according to the codebook, and the codebook is also retrained. Finally, the weights are coded and the indexes are generated using Huffman coding method. 


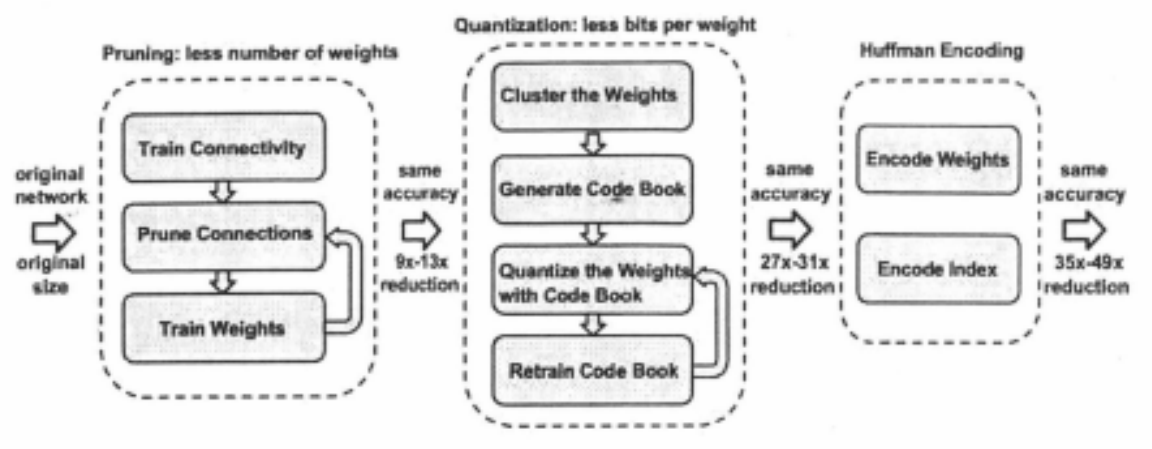

FIG. 2-2 The three-layer pipeline work flow of DeepCOMPRESSION technology

\section{DPM method for model of deformable parts}

The DEformable component model DPM method is a classical order proposed by P.Izensz-Walb in 2008The target detection algorithm is based on the characteristics of each component of the detection target, so it is used in the actual detection It has quite strong robustness. DPM plays a sliding window role in the detection process, which is in the HoG feature group The feature pyramid is run by sliding, and each window is scored during the run. The score is defined by

Deformation cost function and feature matching function are determined together. DPM has become many traditional target detection algorithms The key components of the method, such as image classification, target segmentation and attitude estimation, etc., include: The optimized HOG feature is used to build the feature pyramid, and the sliding window is used to select the candidate region and adopt branches Vector machines are used to make target classification decisions. In addition, in order to improve the robustness of the model, DPM algorithm is introduced Two optimization strategies are proposed: for the multi-view problem of detecting objects, the multi-component strategy is introduced; For the detection of After analyzing object deformation problem, a component feature strategy based on Pictorial Structure is introduced.

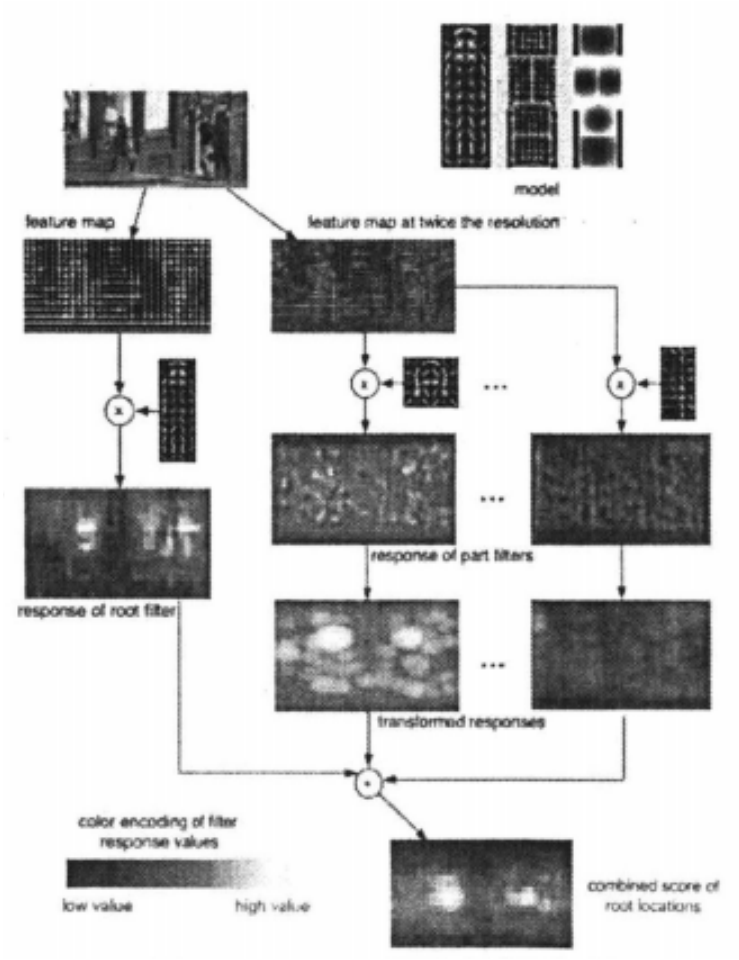

FIG. 3-1 DPM algorithm detection process

\section{Comparative study on the structure of degree neural network}

Based on the deep study of electric power facilities outside the SSD target detection method, outside the power to break the fast detection task, high detection accuracy, can effectively control the power outages due to external force damage caused by the fault, is helpful to reduce the fault treatment and follow-up work of personnel and capital investment, has a positive role on lowering the cost of transmission line operations. This chapter compares the training process, model performance and model generalization ability of SSD method combined with zFNet, VGGNet and $\operatorname{ResNet}(50,101,152)$ with three different structures, and verifies the feasibility of deep learning method in power application scenarios. Experimental results show that in SSD algorithm based on VGGNet network, training convergence speed, the model size is moderate, in fast detection speed and guarantee of the crane, excavator, tower testing higher average 
accuracy of testing on the new data set at the same time average accuracy decreases, less able to identify some fuzzy, obscured the goal, and can do right of various forms of target recognition, generalization ability is strong, more applicable to electric power.

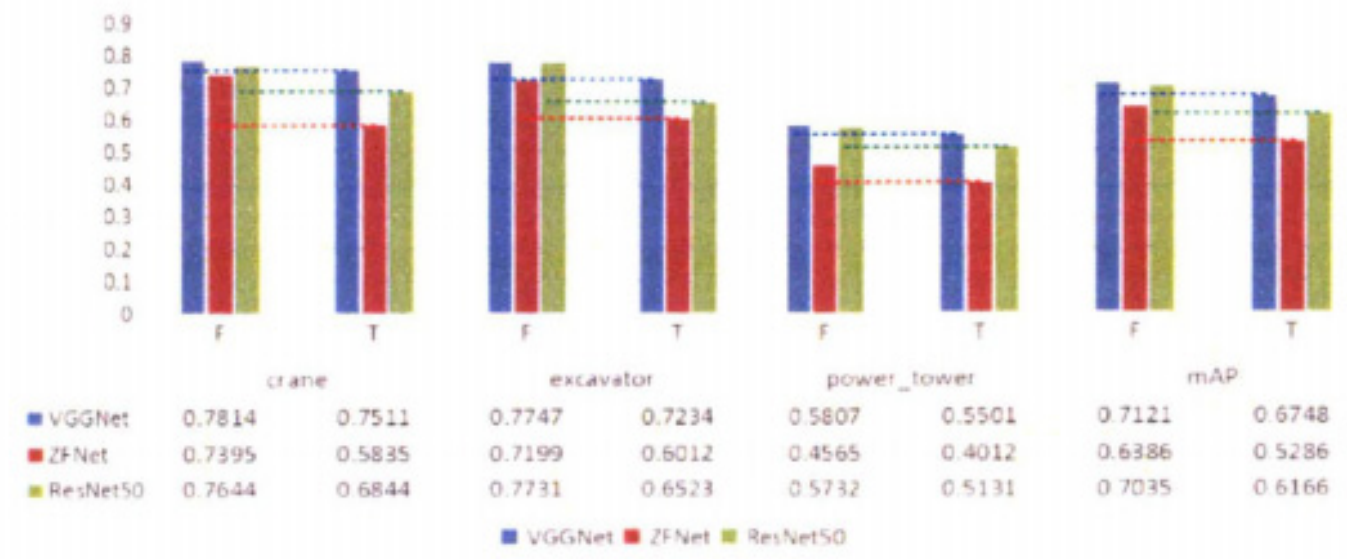

FIG.4-1 Test results of data sets collected on site

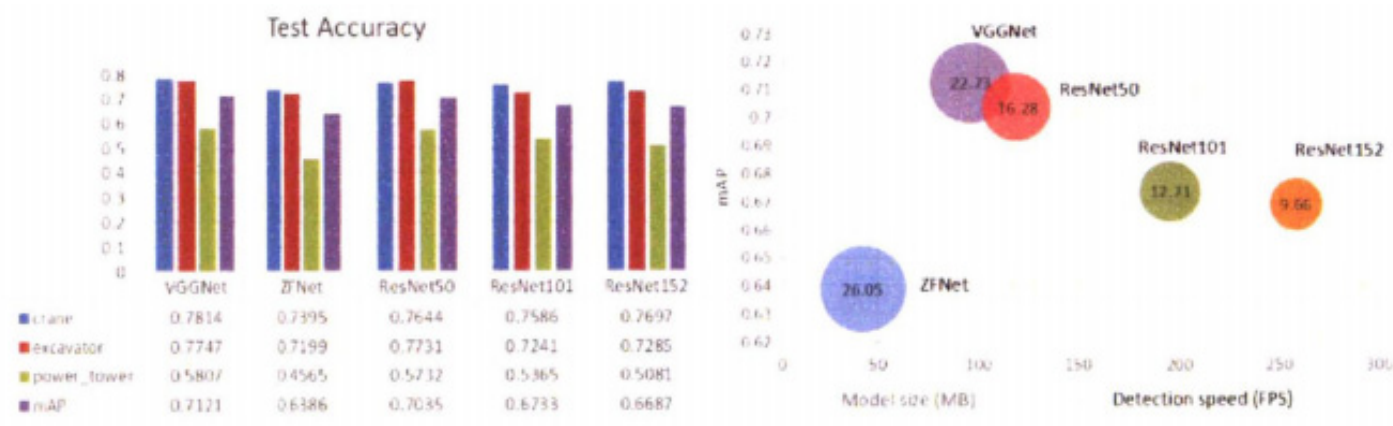

FIG.4-2 Model performance comparison
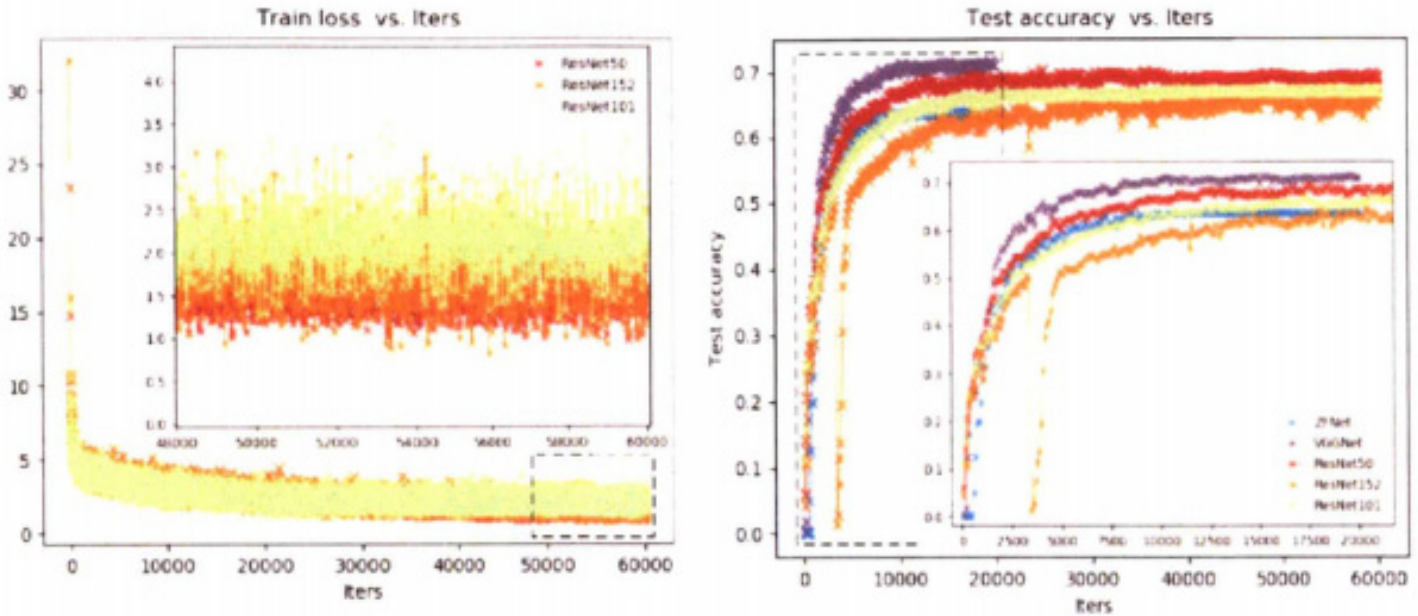

FIG.4-3 Model training process comparison

\section{Conclusion}

Rapid and accurate detection of abnormal conditions in power video surveillance plays a vital role in the safe and stable operation of power grid. Deep learning as a highprofile research direction in the field of machine learning has a great development in recent years, target detection method based on the deep learning by depth of neural networks to target sample of a large number of learning, gradually fitting out the characteristics of high robustness, make more accurate judgment of the target, choice of candidate area is optimized at the same time or even eliminate, greatly accelerated the speed detection, can greatly reduce the artificial labor intensity in electric power inspection, improve work efficiency, laid the security hidden danger in time, complete the troubleshooting. This paper introduces a power vision 
terminal target detection system based on deep learning.

\section{References}

1. N. Dalal and B. Triggs, "Histograms of oriented gradients for human detection,"[C],2005 IEEE Computer Society Conference on Computer Vision and Pattern Recognition (CVPR'05), San Diego, CA, USA, 2005, pp. 886-893 vol. 1.

2. Performance evaluation of texture measures with classification based on Kullback discrimination of distributions [C]. Ojala, T.; Pietikainen, M.; Harwood, D. Source: Proceedings of the 12th IAPR International Conference on Pattern Recognition (Cat. No.94CH3440-5), p 582-5 vol.1, 1994

3. M. Pietikainen, S. Nieminen, E. Marszalec and T. Ojala, "Accurate color discrimination with classification based on feature distributions," [C], Proceedings of 13th International Conference on Pattern Recognition, Vienna. Austria, 1996, pp. 833838 vol.3.

4. O. Penttinen, T. Pyssysalo and L. Ojala, "Performance analysis of adaptive video on demand using stochastic Petri nets," [C]. Proceedings of the Thirtieth Hawaii International Conference on System Sciences, Wailea, HI, USA, 1997, pp. 119-124 vol.5.

5. T. Ojala and M. Pietikainen, "Nonparametric multichannel texture description with simple spatial operators, "[C]. Proceedings. Fourteenth International Conference on Pattern Recognition (Cat. No.98EX170), Brisbane, Queensland, Australia, 1998, pp. 1052-1056 vol.2. 